\title{
LA DIMENSIÓN DE GÉNERO: \\ AUSENTE EN LA REFORMA DE PENSIONES EN MÉXICO*
}

THE GENDER DIMENSION:

ABSENT FROM THE PENSION REFORM IN MEXICO

\author{
LA DIMENSION DE GENRE: \\ ABSENTE DE LA RÉFORME DES RETRAITES AU MEXIQUE
}

María Ascensión MORALES RAMíreZ*

RESUMEN: Las mujeres, en la vejez, manifiestan con especial crudeza la acumulación de las desventajas a las que se enfrentaron durante años. Ellas tienen perspectivas considerablemente más negativas que los hombres con relación al valor de sus pensiones futuras, y son más propensas a pasar sus últimos años de vida en la pobreza. Un conjunto de factores diferenciadores directos e indirectos se concatenan en su perjuicio: las diferencias entre mujeres y hombres en el empleo se recrudecen según el diseño del sistema de pensiones contributivo.

Considerar la dimensión de género en dichos sistemas es una tarea impostergable para erradicar las desigualdades y discriminaciones de las normas que impiden a la mujer obtener una pensión por derecho propio, un retiro digno y una autonomía económica en la vejez.

Palabras clave: género, igualdad de género, sistemas de pensiones, empleo formal, trabajo no remunerado.

ABSTRACT: Women in their old age are particularly hard hit by the accumulation of the disadvantages they have faced for years. They have considerably poorer prospects than men about the amount of their future pensions and are more likely to spend the last years of their lives in

* Recibido el 10 de diciembre de 2020 y aceptado para su publicación el 15 de marzo de 2021.

** Profesora de derecho del trabajo y derecho de la seguridad social en la Facultad de Derecho de la UNAM. 
poverty. A combination of direct and indirect disparities works against them: the differences between men and women in employment are exacerbated by the design of the contributory pension system.

Including a gender dimension in these systems is a matter that can no longer be postponed if we are to eradicate the inequalities and discrimination that prevent women from obtaining a pension, a dignified retirement and economic independence in their old age.

Keywords: gender, gender equality, pension systems, formal employment, unpaid work.

RÉSUMÉ: Les femmes âgées manifestent avec une grossièreté particulière l'accumulation des désavantages auxquels elles ont été confrontées pendant des années. Ils ont des perspectives beaucoup plus négatives que les hommes quant à la valeur de leurs pensions futures et sont plus susceptibles de passer leurs dernières années à vivre dans la pauvreté. Un ensemble de facteurs de différenciation directe et indirecte sont concatenated à leur détriment: les différences entre les femmes et les hommes dans l'emploi sont intensifiées selon la conception du système de pension contributif.

Considérer la dimension sexospécifique de ces systèmes est une tâche impossible pour éradiquer les inégalités et la discrimination des règles qui empêchent les femmes d'obtenir une pension de travail indépendant, une retraite décente et une autonomie économique dans la vieillesse.

Mots-clés: genre, égalité des sexes, systèmes de retraite, emploi formel, travail non rémunéré.

Sumario: I. Introducción. II. Discriminaciones laborales. III. Discriminaciones en los sistemas de pensiones. IV. Experiencias de compensaciones contributivas. V. La reforma mexicana. VI. Conclusiones. VII. Bibliografía.

\section{INTRODUCCIÓN}

$\mathrm{E}$ 1 debate sobre la dimensión de género en los sistemas de pensiones de América Latina ha cobrado gran fuerza en los últimos años. ${ }^{1}$ Los

1 El debate inició en las normas internacionales hace más de seis décadas, con la Declaración sobre la Eliminación de la Discriminación contra la Mujer en 1967 y la Convención 
datos que se proyectan para las próximas décadas respecto de la transición demográfica revelan una feminización del proceso de envejecimiento poblacional en la región.

En 2019, los hombres mayores de 65 años alcanzaron un 7.5\% de la población total de éstos, mientras que la proporción de mujeres de 65 y más años aumentó al 9.4\% del total de ellas. Las proyecciones de población indican que, en 2050, la población de hombres de 65 años y más, representará un $17.6 \%$ del total de ellos, mientras que para las mujeres este porcentaje alcanzará el $21.4 \% .^{2}$

En las reformas paramétricas y estructurales de los últimos 15 años en América Latina, la "dimensión de género" fue considerada un requisito básico y fundamental en el sistema de pensiones, por razones demográficas, de titularidad de derechos y de autonomía económica en la vejez, para hacer frente al conjunto de factores diferenciadores directos e indirectos que generan las desigualdades y discriminaciones entre el hombre y la mujer en el ámbito laboral, así como respecto de la división sexual del trabajo no remunerado, entre otros puntos, los cuales se reproducen y agravan según el diseño y reglas del funcionamiento del sistema de pensiones contributivo, impidiendo el acceso de las mujeres a la protección del sistema y a la obtención de una pensión de vejez.

En México en 2019, a diferencia del camino recorrido por algunos países de la región, la reciente reforma a las pensiones de retiro reguladas en la Ley del Seguro Social (LSS) no se ocupó de la dimensión de género, a pesar de los avances sobre el tema en otros campos y de que, desde hace tiempo, la academia y la sociedad civil habían demandado su inclusión. ${ }^{3}$

Asimismo, dicha dimensión fue ignorada, debido a la pandemia por la COVID-19, cuyos datos señalan que el mayor impacto negativo de esta crisis será en las actividades económicas y en los empleos altamente feminizados.

sobre la Eliminación de Todas las Formas de Discriminación contra la Mujer en 1979, aspectos que fueron incluidos en la seguridad social. La "Recomendación sobre los pisos de protección social" de 2012 confirmó que la seguridad social es una herramienta importante para prevenir y reducir la pobreza, la desigualdad, la exclusión y la inseguridad social, para promover la igualdad de oportunidades, la igualdad de género y la igualdad racial, así como para apoyar la transición del empleo informal al empleo formal.

2 Arenas de Mesa, Alberto, Los sistemas de pensiones en la encrucijada. Desafíos para la sostenibilidad en América Latina, Santiago, CEPAL, 2019, p. 215.

3 Arenas de Mesa, señala que uno de los factores que explica esta situación es la baja participación de las mujeres en la toma de decisiones y en la definición del diseño de los sistemas de pensiones, pues las autoridades de hacienda son hombres. 
La iniciativa presidencial de reforma se presentó el 25 de septiembre de 2019, y fue aprobada en diciembre sin modificación alguna, fast track, por ambas cámaras del Congreso de la Unión, cuyas presidencias están a cargo de mujeres. ${ }^{4}$ El decreto respectivo fue publicado en el Diario Oficial de la Federación el 16 de diciembre de 2020, y el 1o. de enero de 2021 entraron en vigor, parcialmente, algunas disposiciones, porque los cambios principales surtirán efectos hasta 2023.

Grosso modo, la reforma conserva el sistema de capitalización individual (ahorro forzoso) e introduce elementos ajenos a la seguridad social como la Unidad de Medida y Actualización (UMA), y comprendió tres cambios principales en la LSS: reducción de las semanas de cotización y su incremento sucesivo hasta el 2031; incremento gradual y diferenciado de la cuota patronal (2023-2030); recomposición de las aportaciones estatales, modificaciones a los requisitos y monto de la pensión mínima garantizada. ${ }^{5}$ No se profundiza en estos temas aquí, porque existe literatura abundante, ${ }^{6}$ por esto el presente trabajo se centra en examinar las pensiones de vejez de carácter contributivo desde una perspectiva de género ${ }^{7}$ en primer lugar, a partir del análisis de las desigualdades y discriminaciones que enfrenta la mujer en el ámbito laboral (indirectas) y en los sistemas de pensiones (directas). En segundo lugar, a la luz de experiencias que en América Latina han surgido para compensar la falta de cotización con motivo de esos factores diferenciadores. En tercer lugar, se aborda la ausencia de la dimensión de género en la reforma mexicana y, por último, se ofrecen algunas conclusiones.

4 La Cámara de Diputados aprobó la iniciativa presidencial el 8 de diciembre, y la de Senadores el 9 de diciembre de 2020 (en menos de 10 minutos y sin discusión). En 1995, cuando se introdujo el sistema de capitalización individual, la iniciativa presidencial fue presentada el 9 de noviembre, se discutió el 6 y 7 de diciembre, y se publicó el 21 de ese mes en el Diario Oficial de la Federación.

5 Los cambios secundarios fueron las siguientes: modalidades para pensionarse, retiro de las aportaciones voluntarias, imprescriptibilidad para recibir los recursos, entrega de los ahorros de la cuenta individual a los beneficiarios, así como el impacto de los cambios principales en diversos aspectos pensionarios.

6 Cfr. Morales Ramírez, María Ascensión, Reforma en materia de pensiones y la necesidad de crear el sistema nacional de pensiones, México, UNAM, Instituto de Investigaciones Jurídicas, Serie Opiniones Técnicas sobre Temas de Relevancia Nacional, núm. 40, 2021.

7 Proceso de evaluar las implicaciones para mujeres y hombres de cualquier acción planificada, con el fin de que se convierta en una dimensión integral del diseño, implementación, monitoreo y evaluación de políticas y programas. Abarca la capacidad de anticipar el impacto potencialmente diferenciado de las acciones de política sobre mujeres y hombres, así como la capacidad de diseñar acciones políticas que no son ciegas sino sensibles al género. 


\section{DISCRIMINACIONES LABORALES}

\section{Inserción laboral}

La mujer, al participar en el trabajo remunerado, conquistó la posibilidad de ejercer su derecho a la libertad, a la igualdad de trato y a una ciudadanía plena. Igualmente, logró su independencia económica y nuevos campos de realización.

A pesar de ello, el ingreso de la mujer a la vida laboral no ha estado exento de obstáculos y diferencias que la afectan en un doble sentido: en su participación laboral y en su capacidad para cotizar al sistema de pensiones contributivo.

Además, desde hace décadas, el gran deterioro social y económico producto de la pobreza, desigualdad, informalidad y bajo crecimiento económico en el país, han exacerbado las desigualdades de género, entre otras causas, por los factores evidenciados en 2019, entre los que destacan los siguientes: ${ }^{8}$

a) Inserción. La tasa de participación laboral de las mujeres a nivel nacional fue $44.9 \%$, en tanto la de los hombres fue $77.1 \%$. Así, las mujeres representaban el 39\% de la fuerza laboral total del país.

b) Salario. Más de la mitad percibía hasta dos salarios mínimos, de tal suerte que la diferencia salarial entre hombres y mujeres fue del $-6.6 \% .^{9}$

c) Actividad. Se ocuparon en actividades relacionadas con el comercio $(25.8 \%)$ y en los servicios $(53.2 \%) .{ }^{10}$

d) Desempleo. El 55.1\% de las mujeres económicamente activas de 15 y más años no tuvieron acceso a un empleo formal. De ellas, los porcentajes más altos fueron en los grupos de 15 a 19 años (76.5\%) y de 60 y más años $(72.3 \%)$.

e) Empleo informal. El 57.6\% se ocupó en este tipo de empleos, mientras que la tasa de los hombres fue de $55.3 \% .^{11}$

8 INEGI, Mujeres y hombres en México 2019, México, 2019, pp. 106-109.

9 Primera penalización salarial y, por ende, desigualdad de los ingresos asociadas al género.

10 Fenómeno conocido como segregación horizontal, en donde las mujeres se concentran en ocupaciones consideradas "femeninas".

11 México ¿cómo vamos?, Las mujeres en el mercado laboral mexicano, México, 2021, p. 5, disponible en https:// mexicocomovamos.mx/new/md-multimedia/1583538116-995.pdf. 
f) Servicios de guarderías. El $77.7 \%$ de las mujeres con empleo formal no disponían del servicio de guardería o cuidados, únicamente el $22.3 \%$ tienen acceso a estos servicios. ${ }^{12}$

Lo anterior confirma que las mujeres participan menos y de manera más intermitente en el empleo formal, suelen ubicarse en trabajos peor remunerados e inestables, se insertan con mayor probabilidad en la informalidad porque les permite más flexibilidad para atender responsabilidades de su rol tradicional y reciben ingresos menores, producto de la discriminación laboral o de la segregación ocupacional.

La literatura en la materia señala que estos obstáculos han dificultado que las mujeres puedan desarrollar todo su potencial en el trabajo remunerado y, en específico, las madres trabajadoras cuyas edades oscilan entre 25-49 años quienes representan las tasas más bajas en el empleo, con lo que se evidencia que, si bien la inserción laboral femenina ha evolucionado, también lo ha hecho de manera diferente entre grupos de edad, educación ${ }^{13}$ y estado civil. ${ }^{14}$

Asimismo, dichas desigualdades y diferencias en el acceso al empleo (trabajo formal e ingresos) se han trasladado en forma específica a los sistemas de pensiones como desigualdades de cobertura contributiva (por las interrupciones laborales); brecha salarial y menor tiempo de cotización, las cuales, al concluir el ciclo laboral, dificultan a la mujer cumplir con los requisitos de cotización o, aun cumpliéndolos, de cualquier forma, sus pensiones por derecho propio $^{15}$ son de un monto menor.

Esto significa que además de las inequidades heredadas del ámbito laboral a los sistemas de pensiones contributivos, las reglas o diseño de dichos sistemas potencian estas diferencias en las prestaciones y, por tanto, reproducen e incrementan la desigualdad de género.

La pandemia vino a aumentar los niveles de desigualdad de la mujer porque los sectores productivos en donde se inserta tuvieron un impacto negativo mayor. En mayo de 2020, su tasa de participación laboral fue del 43\% y la de los hombres de $66.2 \%$, la tasa de desempleo del $24.6 \%$ en comparación

\footnotetext{
12 INEGI, Mujeres y hombres..., 2019, op. cit, p. 115.

$13 \mathrm{El}$ incremento del nivel educacional femenino aumentó la participación de la mujer en la fuerza laboral.

14 Cfr. Ciedess, Cerrando las brechas de género: es hora de actuar, Santiago, CISS-OCDE, 2014.

15 Una parte importante de las mujeres reciben pensiones por derecho derivado, bajo el enfoque familiar tradicional, consistente en la pensión de viudez, cuyo porcentaje depende del salario del cónyuge fallecido.
} 
con el 17.4\% de los hombres. La Comisión Económica para América Latina (CEPAL) estimó una caída del producto interno bruto (PIB) del 9\% para el país, que significará un retroceso de más de diez años en su participación. ${ }^{16}$

\section{División sexual del trabajo}

La persistencia de los roles tradicionales de género sigue limitando las oportunidades de las mujeres para participar en el trabajo en igualdad de condiciones con los hombres.

Así, la menor o intermitente participación de la mujer en el ámbito laboral está relacionada con sus responsabilidades biológicas de la maternidad, el cuidado de los hijos o de las personas mayores y/o con discapacidad, así como las tareas del hogar, esto es, con la carga del trabajo no remunerado, la distribución desigual de las responsabilidades del hogar y el cuidado de los dependientes, por esto, la literatura en la materia afirma que las mujeres dedicaban el triple de tiempo que los hombres a estas actividades.

En efecto, en el país las mujeres destinan al trabajo no remunerado 39.1 horas a la semana y los hombres 14.1, esto implica que por cada hora que ellos destinan, ellas realizan aproximadamente tres, aunque la participación por tipo de actividad realizada de unas y otros suele tener variaciones. ${ }^{17} \mathrm{La}$ Cuenta Satélite del Trabajo no Remunerado, a lo largo del periodo 2010 a 2017, consideró que el trabajo no remunerado equivalía a una quinta parte del PIB. ${ }^{18}$

La literatura al respecto afirma que, con estas interrupciones, la brecha salarial de género aumenta de manera abrupta, lo cual indica que, en la cultura del trabajo, la maternidad y crianza de los hijos y los cuidados se "penaliza" con una pérdida salarial importante. ${ }^{19}$

A pesar del cambio de paradigma en los estándares tradicionales de género y el aumento de las familias monoparentales, que han propiciado la mayor incorporación de las mujeres en el trabajo, las actividades de su rol tradicional

16 CEPAL, "La autonomía económica de las mujeres en la recuperación sostenible y con igualdad”, Informe Especial COVID-19, núm. 9, febrero de 2021, pp. 2 y 3.

17 INEGI, Mujeres y hombres..., op. cit., p. 124.

18 Durante 2017, el valor económico del trabajo no remunerado ascendió a 5.1 billones de pesos valorados a precios corrientes, es decir, el 23.3\% del PIB de ese año. Del citado valor, tres cuartas partes fueron aportadas por las mujeres y el resto por los hombres

19 Jiménez Lara, Antonio et al, Estudio sobre la perspectiva de género en los sistemas de seguridad social en Iberoamérica, Madrid, OISS, 2013. 
continúan a su cargo. Por una parte, esto se debe a la división sexual del trabajo y, por otra, ante las insuficientes políticas públicas y la segmentada oferta del mercado de servicios de cuidado. Sin embargo, en ambos casos, ante las interrupciones laborales para dedicarse a esas actividades, las mujeres enfrentan lagunas de protección que más tarde influyen en las pensiones de vejez, dado que sus aportaciones se verán estancadas y/o reducidas y, por ende, en el derecho a adquirir esa prestación. ${ }^{20}$

\section{Diferencias biodemográficas}

Los antecedentes demográficos indican que las mujeres tienen una esperanza de vida mayor que los hombres. En 1990, en el país se esperaba que una recién nacida viviera en promedio 73.9 años. En 2019, se estimó la expectativa de vida de la mujer en 78 años y para el 2030 en 79.6 años. La diferencia por sexo era de 6 años menos para los hombres en 1990 (68 años), se mantuvo en 2019 y se espera igual en 2030.21 Así, la transición demográfica tiene un carácter femenino.

Según datos de la Encuesta Nacional de la Dinámica Demográfica (Enadid) 2018, las personas mayores en el país eran 15.4 millones, cifra que representaba el $12.3 \%$ de la población total. De esta cantidad, más de 8 millones son mujeres mayores.

Así, las mujeres, además de envejecer a una tasa más alta, también representan un porcentaje mayor dentro de las personas mayores. Estas dos condiciones se traducen, a los sistemas de pensiones, en una menor siniestralidad durante la vida laboral activa de las mujeres y un periodo de vejez más extenso para financiar sus pensiones de vejez.

Por esto, algunos sistemas de pensiones, sobre todo aquellos cuyo método de financiamiento es de capitalización individual (como el mexicano), consideran las tablas de mortalidad diferenciadas por sexo, las cuales afectan el valor de las prestaciones a las que pueden acceder las mujeres, para un mismo esfuerzo contributivo.

En efecto, el uso de tablas de mortalidad diferenciadas basadas en la esperanza de vida produce un impacto negativo para las mujeres, porque al concluir su vida laboral tendrán una pensión menor y durante un tiempo mayor, con lo que se afecta su calidad de vida en el largo plazo.

20 La CEPAL señala que el trabajo no remunerado de los cuidados sigue estando invisibilizado, subestimado y desatendido.

21 INEGI, Mujeres y hombres en México..., op. cit., p. 6. 
El argumento que está detrás del uso de las tablas de mortalidad diferenciadas es que permiten un beneficio equivalente durante el resto de su vida a hombres y mujeres, evitando que el grupo de contribuyentes de aquéllos subsidie al grupo de éstas, cuya expectativa de vida es superior y su permanencia en el sistema de pensiones también lo será. ${ }^{22}$

En el caso mexicano, al continuar con el sistema de capitalización individual y no haberse previsto ninguna disposición en favor de las mujeres, se correrá el riesgo de que las aseguradoras apliquen las tablas de mortalidad diferenciadas en perjuicio de la feminización del proceso de envejecimiento.

\section{DISCRIMINACIONES EN LOS SISTEMAS DE PENSIONES}

El diseño de los sistemas de pensiones contributivos tiene una incidencia directa en la profundización o mitigación de las desigualdades de género. En su origen, dichos sistemas fueron creados sobre la base de un modelo en donde el hombre era el proveedor, el sostén de una familia, contaba con un empleo formal y estable. Así, en todos los países, los derechos pensionarios en distinto grado fueron vinculados a la historia laboral masculina, a sus ingresos y a sus cotizaciones al sistema.

La incorporación de la mujer en la vida laboral vino a evidenciar que los sistemas de pensiones no son neutros: el diseño y reglas de funcionamiento agravan las desigualdades y discriminaciones de género enfrentadas por las mujeres en múltiples ámbitos de la vida social y económica. Además, el diseño de algunos sistemas pensionarios resulta más perjudicial que otros para el sector femenino.

En el sistema de pensiones tradicional de reparto, las cotizaciones de los trabajadores activos se destinan a financiar las pensiones existentes en ese momento, por ello se conoce como de solidaridad intergeneracional, así sus características son beneficios definidos, solidaridad intergeneracional y administración pública. En este sistema, la pensión depende de los salarios de toda la vida o los mejores años o años finales.

Dicho sistema genera transferencias solidarias entre géneros, sin embargo, pueden presentar desigualdades de género en dos aspectos: a) al determinar las pensiones con base en toda la carrera laboral, y b) al establecer una edad de retiro diferenciada, sin compensación alguna. ${ }^{23}$

22 Arenas de Mesa, Alberto, op. cit., p. 217.

23 La literatura en la materia señala que al permitir a la mujer un retiro más temprano, se reduce el monto de la pensión entre un 30\% o $40 \%$. 
En el sistema de capitalización individual las pensiones guardan una relación directa con las aportaciones realizadas (menos las comisiones cobradas por las administradoras privadas y, en su caso, la existencia de pérdidas derivadas de la gestión de las inversiones o las crisis económicas), así sus características son: ahorro forzoso, beneficios indefinidos y administración privada. En este sistema opera el principio de justicia inherente: cada uno recibe los beneficios acordes a sus cotizaciones. Además, el sistema utiliza tablas de mortalidad diferenciadas por sexo para el cálculo de la prestación. Así, la mujer al cotizar por un periodo menor, sus recursos ahorrados son también menores y el uso de las tablas de mortalidad diferenciadas propician una nula o baja pensión.

Con la oleada de reformas en los años noventa a los sistemas de pensiones contributivos (entre ellos el mexicano), hacia el sistema de capitalización individual, ${ }^{24}$ la desprotección hacia la mujer aumentó porque establece condiciones de adquisición más estrictas. Las normas bajo este sistema están perfiladas hacia un determinado tipo de mujer: pertenece al sector formal de la economía, se emplea joven, tiene formas de resolver el asunto del cuidado de los hijos y permanece en el trabajo por un largo tiempo.

En Chile, país pionero en introducir el sistema de capitalización individual, la realidad evidenció que las discriminaciones indirectas como la densidad inferior de las cotizaciones con relación a la de los hombres, la esporádica participación en el mundo laboral o la interrupción al mismo, el comportamiento procíclico del desempleo femenino, entre otros, dificultaron a la mujer obtener una pensión de vejez, en razón de que el ahorro acumulado resultó ser insuficiente para financiar esta prestación.

Con lo anterior, se confirmó que el paradigma inspirado en la idea de la gestión individual del riesgo social se topó con obstáculos que impiden su cabal desarrollo: alta informalidad, desempleo, bajos salarios e incremento en la inequidad de género. Tales elementos constituyeron algunas razones de las denominadas "re-reformas" (Chile) y contrarreformas (Argentina y Bolivia) experimentadas después del primer lustro del siglo XXI ${ }^{25}$ en los sistemas de pensiones de capitalización individual en algunos países de la región, con miras a enfrentar, entre otros aspectos, la problemática de género.

24 Además de la modificación fiscal del cambio de sistema, se esperaba que la reforma generara beneficios macroeconómicos, como el incremento del empleo y de la productividad, ahorro interno y desarrollo de mercado de capitales, es decir, se priorizaron estos aspectos en lugar de los objetivos sociales de los sistemas de pensiones.

25 Mesa-Lago, Carmelo et al., "Re-reformas de sistemas de pensiones privatizadoras en el mundo: Estudio comparativo de Argentina, Bolivia, Chile y Hungría", Revista de Trabajo, México, Centro de Análisis del Trabajo-Plaza y Valdez, núm. 10, 2014. 
En 2006, el gobierno chileno conformó un Consejo asesor presidencial para realizar un diagnóstico del sistema. El informe del Consejo dio cuenta de que la dimensión de género no fue considerada en el diseño del sistema y, por ende, el resultado fue negativo para las mujeres, por un lado, al no haber cotizado los años exigidos y, por otro, en caso de haber cubierto los requisitos, la aplicación de tablas de mortalidad diferenciadas por sexo generaba una pensión menor. ${ }^{26}$

El diagnóstico exhibió los efectos de los factores diferenciadores que enfrentó la mujer. Asumir la maternidad y el cuidado de los hijos condujo con frecuencia a su salida del mercado laboral o dejar de buscar trabajo si no lo tenía u optar por trabajar fuera del hogar a tiempo parcial, situaciones que acarrearon como efectos directos la disminución presente y futura de sus ingresos vía una pensión. Esta situación la ha colocado como trabajadora de segunda clase, tanto por su rápida expulsión del mundo laboral cuando la atención de la familia precisa mayor dedicación, como por el menor valor que se le da. Esta es una de las causas principales por las que muchas mujeres llegan a la vejez sin pensión por derecho propio.

Adicional a este panorama, se encontraba el hecho de que cada vez eran y son más frecuentes los divorcios y ruptura de vínculos de pareja. Esta situación alertó sobre las profundas repercusiones en la seguridad de los ingresos en la vejez de las mujeres divorciadas y separadas, especialmente si no han contribuido personalmente a un programa de pensiones a través del trabajo. En este supuesto, si el exmarido vuelve a casarse, pueden perder todo o parte de su derecho a recibir una pensión de superviviente. ${ }^{27}$

Así el diagnóstico consideró que eran muchos los aspectos que el sistema de pensiones debía tomar en cuenta para corregir todas las desigualdades hacia la mujer, en razón de que las funciones familiares y laborales entre hombres y mujeres han cambiado significativamente en la actualidad, pues es un hecho que cuando los niños crecen y empiezan la enseñanza obligatoria, las madres con frecuencia vuelven a entrar al mercado laboral o cambian un trabajo de medio tiempo por uno de tiempo completo (claro el número de hijos juega un papel importante en las decisiones de las trabajadoras) ${ }^{28}$ o cada vez son más las familias monoparentales, razón por la cual el gobierno chileno en

26 Consejo Asesor Presidencial para la Reforma Previsional, Reforma al sistema de pensiones en Chile, Santiago, Gobierno de Chile, vol. 1.

27 Bertranou, Fabio M., Protección social, pensiones y género, Santiago, OIT, 2003.

28 Las madres con tres o más hijos tienen significativamente menos probabilidades de tener empleo. 
2008 tomó cartas en materia de la interacción entre pensiones e igualdad de género, a través de la inclusión de mecanismos compensatorios, medida que fue considerada por otros países.

\section{EXPERIENCIAS DE COMPENSACIONES CONTRIBUTIVAS}

Las desigualdades de género respecto de la cobertura y beneficios de las pensiones de vejez, producto del desbalance en el empleo y del diseño de los sistemas pensionarios, así como las nuevas estructuras familiares, en especial el aumento de los hogares con proveedoras femeninas e incremento de las separaciones conyugales, desde hace tiempo han puesto de manifiesto los riesgos asociados a las diferencias en el bajo o nulo acceso a los beneficios de las pensiones para las mujeres.

Así, en las últimas décadas, esta tendencia se ha venido revirtiendo a través de reconocer derechos individuales de la mujer, y no sólo aquellos asociados a su rol en el hogar, con el fin de compensar la falta de cotizaciones y garantizar su autonomía económica.

Diversos países han introducido disposiciones en el sistema de pensiones contributivo, ${ }^{29}$ para enfrentar el menor porcentaje de cotización al sistema de la mujer activa (densidad de las cotizaciones) y las interrupciones laborales, mediante diferentes modalidades, entre otras:

a) Otorgar bonificaciones o créditos de pensión por maternidad.

b) Introducir un salario de referencia con perspectiva de género para el cálculo de las pensiones.

c) Reconocer derechos pensionales por el trabajo no remunerado.

d) Compensar la ruptura matrimonial.

e) Eliminar las tablas de mortalidad diferenciadas.

\section{Bonos compensatorios}

En América Latina, los créditos o compensaciones para nivelar a la mujer por las interrupciones en la actividad laboral por el cuidado de los hijos,

29 En el marco de prestaciones no contributivas, se han incorporado medidas mediante la provisión de prestaciones universales. 
tal como si hubieran estado empleadas y cotizando al sistema de pensiones, han empezado a aparecer a partir del primer lustro del siglo XXI. En Europa, prácticamente todos los países regulan diversos tipos de medidas compensatorias. ${ }^{30}$

Costa Rica, en 2005, fue el país pionero en reconocer los créditos por maternidad en la reforma paramétrica de ese año y marcó una nueva tendencia en la región. Otorga un bono que equivale a seis meses de cotizaciones a todas las mujeres que se acojan al retiro temprano, en compensación por las labores del cuidado de los hijos. ${ }^{31}$

Chile, en 2008, incorporó al sistema de pensiones un "subsidio a la maternidad", 32 mediante el cual la mujer tiene derecho a recibir una bonificación monetaria por cada hijo nacido vivo. Dicha prestación equivalente al 10\% de los 18 salarios mínimos vigentes en el mes de nacimiento del hijo y se deposita en su cuenta individual. La bonificación tiene una rentabilidad de un $4 \%$ real anual.

Uruguay, en 2008, incluyó la compensación equivalente a un año de trabajo dentro de los 30 años requeridos para que la mujer obtenga la pensión mínima por cada hijo hasta un máximo de 5 años, ${ }^{33}$ con el fin de facilitar el acceso de las mujeres mediante el reconocimiento de las discontinuidades en el trabajo que provoca el cuidado de los hijos. ${ }^{34}$

Bolivia, en 2010, introdujo una compensación de doce meses de cotizaciones por hijo hasta el límite de 36 para esta prestación (o sea, por tres hijos), con la condición de que la mujer realice 120 cotizaciones y cumpla con la edad de 58 años. ${ }^{35}$

30 Suecia, por ejemplo, otorga una aportación a la madre por un máximo de cuatro años. Puede consistir en: a) una aportación del $75 \%$ del promedio de los ingresos de la economía, b) una aportación del $80 \%$ de las cotizaciones realizadas por la trabajadora en el año anterior al nacimiento del hijo, y c) un monto fijo. Austria, en 2007, concedió un monto mensual por niño hasta los cuatro años. Sólo se consideran dos años por niño para los derechos de pensión. España computa un total de 112 días de cotización por hijo nacido y de 14 días más si el parto fuera múltiple. Cfr. Ministerio de Salud y Asuntos Sociales, El sistema sueco de pensión de jubilación, Estocolmo, 2004. Ciedess, Cerrando las brechas de género..., cit.

31 Ley de Protección al Trabajador.

32 De acuerdo con el informe del Consejo Asesor Presidencial para la Reforma Previsional, el $66 \%$ de las mujeres recibía una pensión inferior a su actividad, en comparación con el 23\% de los hombres.

33 Mesa-Lago, op. cit.

34 Esta prestación se estableció en el 2008 en la Ley 18.395. A partir del 2009, el 30\% de las pensionadas ha hecho uso de este beneficio.

35 Artículo 77 de la Ley 065. 
2. Salario de referencia con perspectiva de género

Con el propósito de disminuir la brecha de género en el monto de la pensión a causa del nivel salarial, se ha considerado un salario de referencia específico para las mujeres en el cálculo de sus pensiones.

Costa Rica, en 2005, reguló como salario de referencia los 48 mejores salarios de los últimos 60 cotizados, considerando el promedio de los 20 últimos años de trabajo.

\section{Reconocimiento pensional del trabajo no remunerado}

Algunos países han incorporado disposiciones para reconocer el trabajo no remunerado en el sistema de pensiones con cargo al cónyuge.

Bolivia, en 2010, estableció la obligación de los trabajadores a pagar cotizaciones a favor de sus cónyuges en caso de que éstas se dediquen a las labores del hogar. ${ }^{36}$

Ecuador, en 2015, incorporó el trabajo doméstico no remunerado al sistema para las pensiones de vejez, invalidez y muerte. ${ }^{37}$ Esto supone que las mujeres dedicadas (total o parcialmente) al cuidado de los hijos y del hogar son consideradas para cotizar en el sistema de pensiones. Las cotizaciones son cubiertas en parte por la familia y en parte por el Estado, y son diferenciadas según el nivel socioeconómico del hogar, ${ }^{38}$ aunque en forma contraria a la Constitución que estableció en el artículo 369, inciso dos, el financiamiento de este tipo de cotizaciones a cargo del Estado.

\section{Tablas de mortalidad unisex para el cálculo de las pensiones}

Con el objeto de contrarrestar la discriminación de género en el sistema de capitalización individual a causa de la mayor expectativa de vida de la mujer con respecto de la de los hombres, algunas legislaciones han incorporado tablas de mortalidad unisex para el cálculo del monto de la renta vitalicia.

36 Pese a que la demanda original, por parte de las mujeres, era que la aportación fuera estatal, porque el éxito de la medida resultaba dudoso.

37 Ley de Seguridad Social, artículo 2o., inciso g), derivado de la reforma a la Ley Orgánica para la Justicia Laboral y Reconocimiento del Trabajo no Remunerado, Suplemento de Registro Oficial, núm. 483, 20 de abril de 2015.

38 Marco Navarro, Flavia, La nueva ola de reformas previsionales y la igualdad de género en América Latina, Santiago, CEPAL, 2016. 
Bolivia, en 2010, introdujo la tabla de mortalidad única para hombres y mujeres para el cálculo de prestaciones en el régimen contributivo y semicontributivo. ${ }^{39}$

El Salvador, en 2017, estableció una metodología igualitaria para hombres y mujeres en el cálculo de la renta programada. ${ }^{40}$

\section{Compensación por ruptura matrimonial}

Los sistemas de pensiones contributivos, en su origen, no consideraron la división o compensación de los derechos pensionarios, no obstante que, durante la vida conyugal, se crean derechos de acuerdo con su pacto implícito para generar ingresos monetarios familiares, los cuales se distribuían al interior del hogar.

Sin embargo, con el avance en el reconocimiento de la autonomía de las mujeres para obtener una pensión por derecho propio, también se ha empezado a reconocer el trabajo del hogar y de los cuidados no remunerados (hijos y/o familiares dependientes) que realizan preferentemente las mujeres, a través de la incorporación de una figura conocida como "reparto de la pensión".

Chile, en 2008, producto de la re-reforma al sistema de pensiones, estableció la "compensación económica en caso de nulidad o divorcio". ${ }^{41}$ Se dispuso que cuando el juez determine la existencia de un menoscabo económico ordenará el traspaso de fondos desde la cuenta de capitalización individual del cónyuge que deba compensar a la cuenta del otro. De no existir tal cuenta, el traspaso se realizará a una cuenta voluntaria que deberá abrirse para tal efecto. Dicho traspaso no podrá exceder del $50 \%$ de los recursos acumulados del cónyuge compensatorio durante el matrimonio. ${ }^{42}$

\footnotetext{
39 Artículo 69 de la Ley 065.

40 Arenas de Mesa, Alberto, op. cit., p. 226.

41 Artículo 80 de la Ley núm. 20.255.
}

42 En Alemania, también existe la división de los derechos pensionarios en cada pilar del sistema. Se suman los puntos de pensiones acumulados por ambos cónyuges durante el matrimonio y cada uno se queda con la mitad del total. En caso de que sólo uno de los cónyuges haya adquirido derechos pensionarios, se crea una cuenta para el otro, a la cual se transfiere la mitad de los derechos adquiridos. En Austria, desde 2005 opera la división de los derechos pensionarios durante los primeros cuatro años del nacimiento de los hijos. El cónyuge que tiene trabajo remunerado puede transferir hasta el $50 \%$ de sus derechos pensionarios a la otra persona que cuida a los hijos, sin tener remuneración alguna. Cfr. Arza, Camila, El diseño de los sistemas de pensiones y la igualdad de género, Santiago, CEPAL, 2017, p. 23. 
Como puede apreciarse, la compensación de contribuciones a través de las bonificaciones varía, según los países, en torno al tiempo adicionado por hijo/a, al máximo de hijos/as que se contemplan, y al progenitor que puede hacer uso de dichas bonificaciones (en algunos casos, la madre y en otros, cualquiera de los padres) además las condiciones bajo las cuales pueden acceder al beneficio (mínimo de años de cotización, obligación de haber dejado de trabajar, entre otras).

Si bien un sector de la literatura señala que las compensaciones de ingresos y reparto de la pensión incentivan los roles tradicionales de género, ${ }^{43}$ también es cierto que otros autores opinan que deben existir, aunque con un carácter temporal, con el fin de equilibrar el reconocimiento por el tiempo dedicado al cuidado y los incentivos al trabajo remunerado, ${ }^{44}$ mientras no se implementen medidas para lograr la equidad laboral, coherentes con el reconocimiento de la autonomía y derecho propio de pensión de la mujer.

\section{LA REFORMA MEXICANA}

Las pensiones de retiro reguladas en la LSS agravan las desigualdades y discriminaciones indirectas que afronta la mujer (baja participación, inserción en trabajos de poca productividad, desarrollo de la carrera laboral en profesiones u oficios mal remunerados en comparación con los hombres, mayor incidencia al desempleo, acceso a menores salarios e incorporación en el empleo informal), las cuales le impiden la protección del sistema y el acceso a estas prestaciones y, peor aún, porque tiene que encarar muchos de los factores criticables al sistema de capitalización individual.

Los datos de 2018, en el ámbito laboral, dieron cuenta que del total de mujeres de 15 años y más, el $42.6 \%$ estaba vinculado a la población económicamente activa (PEA) y $57.4 \%$ no. De las mujeres económicamente activas, en el mismo rango de $73 \%$ tenían al menos un hijo nacido vivo, y $27 \%$ no lo tenían. ${ }^{45}$

En materia de pensiones, en el primer trimestre de 2020, existían 66403123 cuentas individuales, de las cuales el $44 \%$ están activas y el $56 \%$ inactivas (por-

43 Por ejemplo, Gilbert, N., "Introduction: The Challenge of Pension Reform and Gender Equality in Aging Societies”. Gender, Issues, vol. 23, núm. 1, pp. 3-5, 2006: citado por Amarante.

44 Amarante, Verónica et al, La brecha de género en jubilaciones y pensiones. Los casos de Argentina, Brasil, Chile y Uruguay, CEPAL, 2016, p. 13.

45 INEGI, "Estadísticas a propósito del día de la madre (10 de mayo) datos nacionales", Comunicado de Prensa, núm. 243/19, 8 de mayo de 2019. 
que los trabajadores no han realizado cotizaciones). Dentro de las cuentas activas, el $40.7 \%$ correspondían a las mujeres y $59.3 \%$ a los hombres. ${ }^{46}$ Desde el inicio de este sistema, la tendencia ha sido la permanencia de un porcentaje mayor de cuentas inactivas, sobre todo, de las mujeres.

La LSS en el artículo 154 establecía los requisitos para el derecho a obtener la pensión de cesantía en edad avanzada: 60 años de edad, no tener trabajo reumunerado y haber cotizado 1,250 semanas (más de 24 años), y el artículo 162 preveía la pensión de vejez: 65 años de edad y 1,250 semanas de cotización. Las edades son para hombres y mujeres.

Estudios internacionales afirmaron, en su momento, que muy pocas personas alcanzarían pensiones, y éstas serían con tasa de reemplazo entre el 23 y $25 \% .{ }^{47}$ Esta situación obedecía a que los trabajadores no llegarían a cotizar 24 años por la situación del empleo en México, que los obliga a entrar y salir de un trabajo formal, pasar a la informalidad o al desempleo, y en peor condición se encontraban las mujeres.

Por ello, desde diversos foros (académico, sociedad civil y organizaciones sindicales) se demandaba una revisión del sistema a fondo con el propósito de realizar un cambio de lo hecho en 1995, cuando se reformó la LSS (dada la evidencia de los resultados negativos en Chile) y respecto de la primera generación de ese sistema en el país, que muy pocos alcanzarían una pensión y ésta sería de un monto muy bajo; asimismo y, entre otros aspectos, incluir la dimensión de género.

Igualmente, se exigía una reforma integral al sistema de pensiones mexicano para eliminar la fragmentación (existencia de muchos esquemas pensionarios); la heterogeneidad (requisitos y beneficios diferentes en cada sistema); la desigualdad (algunos esquemas proporcionan prestaciones jugosas, con cotizaciones mínimas), y la exclusión $(57.5 \%$ de la población económicamente activa no está protegida).

Era menester evaluar el impacto de estas condiciones a la luz de la perspectiva de género, ${ }^{48}$ así como de las evidencias del impacto negativo que tiene el sistema de capitalización individual en la dimensión de género.

46 CONSAR, "Informe Trimestral al H. Congreso de la Unión sobre situación del SAR", México, enero-marzo de 2020.

47 OCDE, "Estudio de la OCDE sobre los sistemas de pensiones: México, 2016". Azuara, Oliver et al, Diagnóstico del sistema de pensiones mexicano y opciones para reformarlo, Banco Interamericano de Desarrollo, 2019.

48 No se trata de un simple análisis técnico, involucra una serie de cuestiones, discusión, principios y objetivos que se pretenden alcanzar en beneficio de la equidad de género. 
$\mathrm{Al}$ respecto, el 22 de julio de 2020, el presidente en forma conjunta con el secretario de Hacienda y Crédito Público, el presidente del Consejo Coordinador Empresarial y el líder de la Confederación Mexicana de los Trabajadores anunció un "Acuerdo de reforma al sistema de pensiones".

A partir de ese día, desde diferentes ámbitos se formularon temas que debía comprender la reforma, entre otros, la dimensión de género; incluso, la iniciativa privada realizó planteamientos para considerar el reconocimiento de semanas de cotizaciones a las mujeres que se separaran del empleo por cuestiones de cuidados infantiles, condicionando la reincorporación posterior al trabajo. Así, la idea era condonar semanas sin cotizar, siempre y cuando se regresara en los siguientes seis meses después del periodo dedicado al hijo recién nacido.49

Sin embargo, cuando el 25 de septiembre de 2020 se presentó formalmente la iniciativa de reforma pensionaria, la "dimensión de género" estuvo ausente, ignorando las desigualdades y discriminaciones tradicionales entre el hombre y la mujer, así como el trabajo remunerado y no remunerado, entre otros aspectos, y sobre todo, las reglas de funcionamiento del sistema de capitalización individual mexicano.

Asimismo, dicha dimensión fue ignorada en momentos en que la triple crisis (sanitaria, social y económica) provocada por la COVID-19, ha agravado y profundizará la desigualdad de género debido a las consecuencia de las medidas adoptadas para frenar el contagio: pérdida de millones de empleos, reducción de salarios y/o pérdida de ingresos, el aumento del desempleo, mayor carga de trabajo doméstico y de cuidados no remunerados, incremento de cuentas individuales inactivas, retiros parciales por parte de los trabajadores de los ahorros depositados en dichas cuentas para autofinanciarse su desempleo, todo ello, con un impacto mayor para las mujeres y los sectores económicos en donde suelen insertarse (comercio y servicios).

El Censo de Población y Vivienda 2020 dio cuenta de las personas que residen en el país: 126014 024. De ellas, el 51.2\% son mujeres (64 540 634). Asimismo, conforme a dicho censo, 62 de cada 100 personas de 12 años y más son económicamente activas. La tasa de participación económica es de 75.8 en hombres y 49.0 en mujeres. ${ }^{50}$

49 AMAFORE, "Reforma a pensiones corrige las deficiencias para lograr mejores condiciones de retiro para los trabajadores: Amafore”, 22 de julio de 2020. Consejo Coordinador Empresarial, Acuerdo para el fortalecimiento de las pensiones de los trabajadores del sector privado, México, julio de 2020.

50 INEGI, "En México somos 126014024 habitantes: Censo de Población y Vivienda 2020”, Comunicado de Prensa, núm. 24/21, 25 de enero de 2021. 
Lo anterior significa que continuarán pesando y reproduciéndose las desigualdades directas e indirectas para las mujeres. En el ámbito del actual sistema de pensiones de capitalización individual pudieron mitigarse esos efectos al mejorar las reglas a su favor, mínimo en tres aspectos: a) mediante la introducción de los bonos para compensar las cotizaciones de la mujer al sistema por el cuidado de los hijos (12 meses por hijo hasta un máximo de tres), b) una aportación estatal (conforme al nivel socioeconómico) para las mujeres dedicadas total o parcialmente a las labores del hogar, a fin de que puedan acceder a una pensión, y c) el uso de las tablas de mortalidad unisex en este sistema.

La interacción entre pensiones y género obligaba en la reciente reforma a considerar dicha dimensión, por ello, continuará como tarea pendiente establecer medidas oportunas antes de la vejez y del retiro de la fuerza de trabajo, que equiparen las bajas aportaciones de las mujeres (con motivo de las actividades reproductivas, del cuidado de los hijos y/o personas mayores, y del hogar), la cotización a las pensiones y, de esta forma, combatan las lagunas de cobertura contributiva, con miras a la obtención de una mejor pensión.

\section{CONCLUSIONES}

No queda la menor duda que el conjunto de diferencias y obstáculos enfrentados por las mujeres deben ser reconocidos por la seguridad social y, específicamente, por los sistemas de pensiones. En este rubro, la igualdad de género va más allá de un trato igualitario para el hombre y la mujer en sentido formal.

El cambio más importante tiene que ver con el nuevo papel de la mujer en el trabajo, en donde la dimensión de género es un tema en el que se debe avanzar y consolidar en el corto plazo.

En los últimos años, la tendencia en las normativas nacionales ha sido el reconocimiento del cuidado de los hijos mediante bonos o compensación de cotizaciones, la regulación del trabajo doméstico y su incorporación a la seguridad social, ${ }^{51}$ así como del trabajo no remunerado, erradicación de las tablas de mortalidad diferenciadas por género, cambios en los cálculos del monto y los requisitos de las pensiones (con una orientación hacia las mujeres).

Incorporar los mecanismos compensatorios de contribuciones constituyen una parte importante, dentro de una necesidad más amplia con enfoque

51 En México, también ya se incluyó la obligación, para los patrones de las personas trabajadoras del hogar, de inscribirlas al IMSS, y pagar las cuotas correspondientes conforme a las normas aplicables en la materia. 
de género, en los sistemas de pensiones. Dichos mecanismos deben ser proporcionales a la magnitud de la diferenciación, sin distorsionar el empleo y sin desincentivar el trabajo remunerado.

En el caso mexicano, continuarán pendientes estos temas, así como el de las guarderías suficientes y asequibles, entre otros, que ayuden a conciliar la vida laboral y familiar a fin de eliminar una de las principales barreras a las oportunidades del empleo de la mujer, conscientes de que una mayor distribución del trabajo remunerado y no remunerado entre mujeres y hombres requiere tiempo, porque implica un cambio de normas, cultura, mentalidad y actitudes.

\section{BIBLIOGRAFÍA}

AMAFORE, "Reforma a pensiones corrige las deficiencias para lograr mejores condiciones de retiro para los trabajadores: Amafore", 22 de julio de 2020.

AMARANTE, Verónica et al., La brecha de género en jubilaciones y pensiones. Los casos de Argentina, Brasil, Chile y Uruguay, CEPAL, Santiago, 2016.

Arenas de Mesa, Alberto, Los sistemas de pensiones en la encrucijada. Desafios para la sostenibilidad en América Latina, Santiago, CEPAL, 2019.

ARZA, Camila, El diseño de los sistemas de pensiones y la igualdad de género, Santiago, CEPAL, 2017.

BerTRANOU, Fabio M., Protección social, pensiones y género, Santiago, OIT, 2003.

CEPAL, "La autonomía económica de las mujeres en la recuperación sostenible y con igualdad", Informe Especial COVID-19, núm. 9, febrero de 2021.

CIEDESS, Cerrando las brechas de género: es hora de actuar, Santiago, CISS- OCDE, 2014.

¿Cómo vamos?, Las mujeres en el mercado laboral mexicano, México, 2021.

CONSAR, "Informe trimestral al H. Congreso de la Unión sobre situación del SAR”, México, enero-marzo de 2020.

Consejo Asesor Presidencial para la Reforma Previsional, Reforma al sistema de pensiones en Chile, Santiago, Gobierno de Chile, vol. 1.

CONSEJO COORDINADOR EMPRESARIAL, Acuerdo para el fortalecimiento de las pensiones de los trabajadores del sector privado, México, julio de 2020. 
INEGI, Mujeres y hombres en México 2019, México, 2019.

INEGI, "Estadísticas a propósito del día de la madre (10 de mayo) datos nacionales", Comunicado de Prensa, núm. 243/19, 8 de mayo de 2019.

INEGI, "En México somos 126014024 habitantes: Censo de Población y Vivienda 2020”, Comunicado de Prensa, núm. 24/21, 25 de enero de 2021.

JimÉNEZ LARA, Antonio et al., Estudio sobre la perspectiva de género en los sistemas de seguridad social en Iberoamérica, Madrid, OISS, 2013.

MARCo NAVARro, Flavia, La nueva ola de reformas previsionales y la igualdad de género en América Latina, CEPAL, Santiago, Serie Asuntos de Género, 2016

MeSA-Lago, Carmelo et al., "Re-reformas de sistemas de pensiones privatizadoras en el mundo: Estudio comparativo de Argentina, Bolivia, Chile y Hungría", Revista de Trabajo, núm. 10, México, Centro de Análisis del Trabajo-Plaza y Valdez, 2014.

Ministerio de ECONOmía y FinanZas Públicas, Boletín Sistema Integral de Pensiones, La Paz, 2013.

Ministerio de SAlud y Asuntos Sociales, El sistema sueco de pensión de jubilación, Estocolmo, 2004.

Morales Ramírez, María Ascensión, Reforma en materia de pensiones y la necesidad de crear el sistema nacional de pensiones, México, UNAM-Instituto de Investigaciones Jurídicas, Serie Opiniones Técnicas sobre Temas de Relevancia Nacional, núm. 40, 2021.

\section{Legislación}

Ley de Protección al Trabajador, Diario Oficial, San José, 2005.

Ley 20.255/2008, Diario Oficial, Santiago, 17 de marzo de 2008.

Ley 26.425, Boletin Oficial, Buenos Aires, 9 de diciembre de 2008.

Ley 065, Gaceta Oficial, La Paz, 10 de diciembre de 2010. 